\title{
Highly ammonia-sensing using direct in situ electro- deposited polypyrrole-dodecylbenzene sulfonic acid film on ITO coated flexible substrates.
}

\author{
Ahmed Mekki1, Fateh Merdj ${ }^{1}$, Djamil Guettiche ${ }^{1}$, Boualem Mettai1 \\ 1 Ecole Militaire Polytechnique, BP 17 Bordj El Bahri 16046, Alger, Algérie \\ Corresponding author: mekki_ahmedkarim@yahoo.fr
}

\begin{abstract}
:
Air quality monitoring is of major concern, as it is directly linked to public health. It requires the development of high sensitive devices with fast response towards hazardous gas and volatile compounds. Such performances depend on the nature and quality of deposition of the sensing layer. Herein, adherent Polypyrrole-dodecylbenzene sulfonic acid (PPy-DBSA) films were deposited on $\mathrm{N}$ (3-trimethoxysilylpropyl) pyrrole modified ITO coated polyethylene terephthalate (PET) flexible substrate by facile direct electrochemical oxidation of pyrrole in an aqueous solution of dodecylbenzene sulfonic acid (DBSA). Chemiresistive gas sensing tests have demonstrated selectivity and sensitivity of films toward ammonia vapors over the other vapors (nitrogen dioxide, carbon dioxide, hydrogen sulfide, acetone, methanol and ethanol) with higher response at $20 \mathrm{ppm}$, reasonably fast response time of $3 \mathrm{~min}$ and reaching detection limit of $3 \mathrm{ppm}$. The response of the sensor could be related to the strong electrostatic interactions between vapor molecules and the dopant agents within PPy films.
\end{abstract}

Key words: Polypyrrole/ DBSA films, electropolymerization, flexible chemiresistive ammonia sensor.

\section{Introduction}

The detection of ammonia gas at a very low level of concentration is considered as defiance in the field of gas sensors development. Owing to its potential effects when its concentration exceeds the threshold limit value of $25 \mathrm{ppm}$, particularly on the human respiratory system, causing severe irritation of the nose and throat, inducing cough, breathing difficulties and tightness in the chest, moreover, it may irritates or burns the eyes, resulting the blindness. This has therefore created needs for fast, reliable, cost effective and highly sensitive systems for the detection of $\mathrm{NH} 3$ across industrial, commercial and domestic areas. Researches' efforts have been extended to the investigation of sorbent materials, particularly polymers thin films and their composites, which can satisfy good sensing requirements. Amongst the various tested polymer categories, inherently conductive polymer-based chemiresistive gas sensor have shown promises applications. Polypyrrole is widely sought-after, due to its excellent stability under environmental conditions, good conductivity and biocompatibility, it was one of the polymers widely used in alcohols and other organic vapors sensing. [1].

In the present work, we try to portray, the sensing behavior of electrodeposited polypyrrole/DBSA films on Indium-Tin-Oxide coated polyethylene terephthalate (ITO/PET) and treated on its surface through covalent grafting of $\mathrm{N}$-(3-trimethoxysilylpropyl) pyrrole.

Surface modification of ITO/PET substrate and electrodeposition of polypyrrole-DBSA thin layer

ITO-coated PET substrates were hydroxylated by dipping in the potassium hydroxide (KOH) containing dimethyl- sulphoxide (DMSO) solution during 30 minutes, after that, they were dipped in a $\mathrm{N}$-(3-trimethoxysilylpropyl) pyrrole solution and left to react for $48 \mathrm{hr}$.

The electro-polymerization of pyrrole on silanized ITO/BOPET sheets was carried out at room temperature. All potentials were referred to a silver electrode $(\mathrm{Ag} / \mathrm{AgCl})$, the electrolyte solution of pyrrole monomer and DBSA was deaerated by a dry nitrogen stream and 
maintained covered by an aluminum foil to be sheltered from light during the experiments. Upon starting the process, two cycles were registered as shown on Figure. 1 from an initial potential, $\mathrm{Ei}$, of $0.0 \mathrm{~V}$ to a final potential, Ef, of $+1.4 \mathrm{~V}$ at a scan rate of $20 \mathrm{mV} / \mathrm{s}$ for 2 cycles.

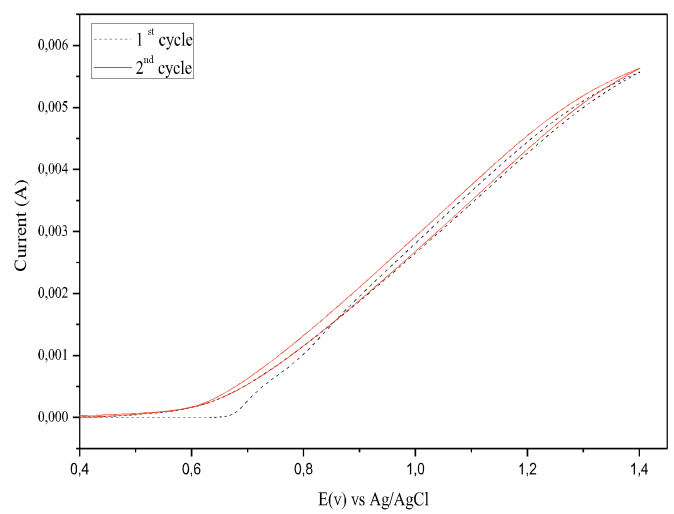

FIG.1: Cyclic voltammogram recorded between 0.0 and $1.4 \mathrm{~V}$.

\section{Chemiresistive gas sensing characteristics}

All vapor tests were performed at temperature of about $25^{\circ} \mathrm{C}$. The concentration of injected analytes was determined in part per million (ppm) according to the following equation (1) described in our previous work [2].

$C=\frac{22.4 \rho T V_{s}}{273 M V} \times 10^{-3}$

Where $C$ is the concentration given in ppm, $\rho$ is the density of liquid sample expressed in $\mathrm{g} / \mathrm{ml}$, $T$ is the cell detection temperature given in Kelvin, $V s$ is the volume of liquid sample in $\mu \mathrm{L}$, $\mathrm{M}$ is the molecular weight of sample in $\mathrm{g} / \mathrm{mole}$, and $V$ is the cell volume expressed in liter. Surface recovering of the flexible samples was insured by flushing thoroughly the test chamber with hot nitrogen. The backshift to the initial value of the sample was considered as an indication of full desorption. The response time is determined as the time corresponding to $90 \%$ of the total change in conductivity upon exposure to a given concentration of organic vapors.

Sensing results related to the selectivity test of different vapors composed of ammonia, nitrogen dioxide, hydrogen sulfide; acetone, ethanol, methanol, and carbon dioxide, injected at room temperature are presented on Figure. 2. PPy-DBSA film showed high response toward $\mathrm{NH}$. On the bar graphs depicted on figures 2 , it is observed that the sensor exhibited less response to the other target formed of reducing gases such as $\mathrm{H} 2 \mathrm{~S}$, $\mathrm{C} 2 \mathrm{H} 5 \mathrm{OH}, \mathrm{CH} 3 \mathrm{OH}$, and oxidizing gas such as $\mathrm{NO} 2, \mathrm{CO} 2$, and $\mathrm{C} 3 \mathrm{H} 6 \mathrm{O}$. It is perceptible that the films response is oriented in its high rate to the reducing vapors of ammonia $\mathrm{NH}$. This may be resulted from the electrostatic interaction generated between the PPy-DBSA structure and the ammonia NH3vapor. This is mostly related to the effect from the dopant agent DBSA structure used within the PPy, which is expected to develop more hydrogen bonds, particularly raised from the presence of sulfonyl groups of the DBSA dopant structure.

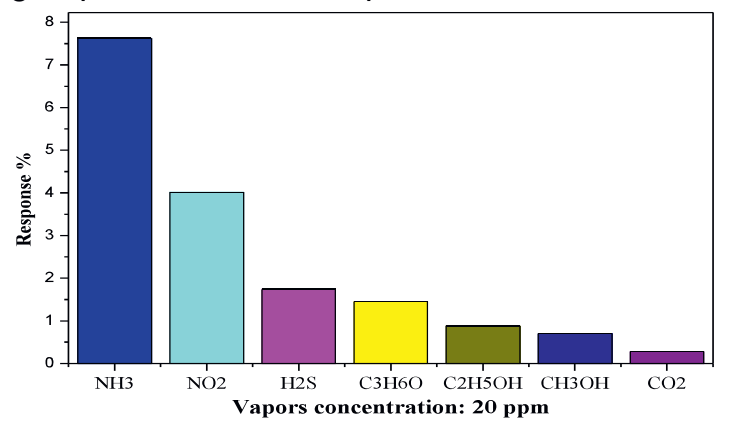

FIG.2: Response of PPy/DBSA films toward vapors at concentration of $20 \mathrm{ppm}$.

The sensors were exposed to different concentrations of $\mathrm{NH} 3$ gas in dynamic injections in the 5-40 ppm range as shown figure 3. Different rates of stepwise increase in response were observed when increase ammonia gas concentration.

The sensors were exposed to different concentrations of $\mathrm{NH} 3$ gas in dynamic injections in the 5-40 ppm range as shown figure 3. Different rates of stepwise increase in response were observed when increase ammonia gas concentration.

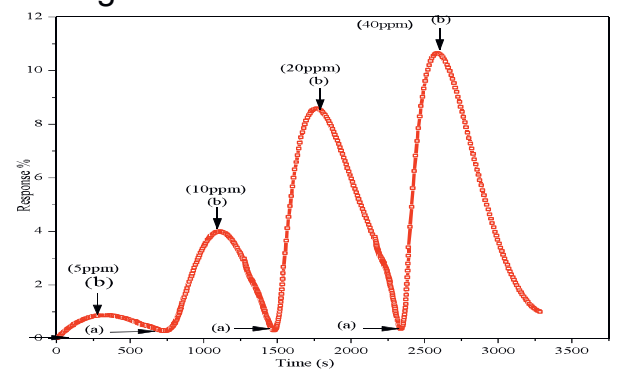

FIG.3: Dynamic sensors response.

\section{References}

[1] T. Björn, W. Olthuis, A.V.D Berg. "Ammonia sensors and their applications-a review. Sensors and Actuators B: Chemical 107,666-677 (2005); doi: 10.1016/j.snb.2004.11.054

[2] Z Ihdene,, A. Mekki, B. Mettai,, R. Mahmoud, B. Hamada, M.M. Chehimi, Quartz crystal microbalance VOCs sensor based on dip coated polyaniline emeraldine salt thin films, Sensors and Actuators B: Chemical 203, 647-654 (2014); doi: doi.org/10.1016/j.snb.2014.07.030 